This is a post-peer-review, pre-copyedit version of a paper published in Advances in Mechanism and Machine Science. IFToMM WC 2019. Mechanisms and Machine Science 73 : 785-794 (2019). The final authenticated version is available online at: http://dx.doi.org/10.1007/978-3-030-20131-9 78.

\title{
Optimum dimensional synthesis using GIMSYNT software
}

\author{
Mónica Urízar ${ }^{10000-0002-8931-6401]}$, Aitor Muñoyerro ${ }^{1}$ Enrique Amezua ${ }^{1[0000-0001-8043-3575]}$ \\ and A. Hernández ${ }^{1[0000-0001-5475-6960]}$ \\ ${ }^{1}$ Faculty of Engineering in Bilbao, University of the Basque Country (UPV/EHU), Spain \\ monica.urizardehu.es
}

\begin{abstract}
In the field of mechanism design, optimum dimensional synthesis plays a significant role. Focusing on path generation synthesis, the designer has to find the most adequate mechanism capable of tracing a trajectory as similar as possible to a prescribed one. The objective is obviously clear, but, in many occasions, the methods dealing with optimum synthesis are quite complex and with a lack of transparency. Consequently, students often get lost in the insights of the optimization method and do not comprehend the influence of the different parameters that can be included in the optimization, or important choices such as enhancing the starting mechanism. Thus, they are not qualified to assess the validity of the resulting optimum design. To overcome this lack of knowledge, we propose a didactic optimum dimensional synthesis methodology mainly based on the analytic relations of the mechanism under study and considering the interrelation among synthesis variables. The guidelines of the procedure can be easily programmed and different design criteria can be incorporated. A software named GIMSYNT has been developed with this purpose, focusing on the slider-crank and the four-bar mechanism.
\end{abstract}

Keywords: Mechanism Design, Optimum Synthesis, Educational Software

\section{Introduction}

In the field of Mechanical Engineering, in particular in Mechanism and Machine Theory, many of the exercises or problems outlined to the students are not just related to the analysis of a certain given mechanism, but that in many occasions, the students have to design the mechanism to perform a specific task. In general, this type of open problems, meaning that more than one solution exists and some criteria design must be incorporated, generates uncertainty to the students. In this area, many problems in mechanical design, and more particularly in dimensional synthesis of mechanisms, can be included.

Regarding the methods related to exact dimensional synthesis, graphical methods to design a planar mechanism that traces a trajectory passing through a maximum of five precision points or positions (for path generation or rigid boy motion respectively) are 
taught to the students. In many universities, these themes are taught during the Bachelor degree, and there exist some softwares, as for example GIM [1], intended for helping the students to better comprehend the theoretical concepts. When more precision points are required and the mechanism does not have as many design parameters, the only option is to proceed with optimum synthesis methods, which obtain approximate solutions.

Dealing with optimization techniques numerous approaches can be found, some of them particularized for certain mechanisms, such as the four-bar mechanism, and other ones of general purpose. Their common target is to minimize a certain function that assesses the mechanism's capacity to accomplish the desired synthesis. Basically, two main groups can be distinguished: local and global methods. Among local methods, the most effective ones are based on the gradient of the function to be minimized [2-4]. An inconvenient of this type of methods is that, in general, the resulting solution depends greatly on the starting mechanism that is established as the initial guess. Regarding the global methods, those based on genetic algorithm techniques are undoubtedly the most popular and effective ones. They are inspired in biological principles, and based on four stochastic processes to enhance the survival skills: reproduction, mutation, competition and selection. As a reference, one of the most complete and efficient approach is developed in [5]. Additionally, in many occasions both local and global tactics are combined to carry out a hybrid optimization approach [6]. Frequently, the global approach is initially applied to find an adequate starting mechanism, and then, a local optimization method is subsequently used to find the final optimum design. Bearing in mind the importance of the starting design, other procedures based on the comparison among different shapes of the coupler-curves associated with several designs of the mechanism under study are also used [7]. In conclusion, after all these years, dimensional synthesis of mechanisms is still a current issue, in particular many present works dealing with the four-bar mechanism $[8,9]$.

From an educational point of view, these optimization methods, in general, follow a complex process and the students do not get the insights of the approach. As a consequence, they just apply the methods without really understanding the influence of the different choices that can be implemented, and with no capacity of evaluating if the final result could have been improved. With the purpose of overcoming this difficulty, we propose an optimum dimensional synthesis approach built with some basic and ordered guidelines, mainly based on the analytic equations related to the mechanism under study and the dependence among the synthesis variables, that combined with certain design criteria result in an easy-to-follow procedure which helps the students getting deeper into the optimum synthesis. To facilitate the comprehension of the procedure and to evaluate the influence of all the implemented options, GIMSYNT software has been created, its main capacities being presented in this paper. 


\section{Procedure for optimum dimensional synthesis}

The basic guidelines of the procedure for optimum dimensional synthesis are the following: first, the relation among the variables or parameters involved in the synthesis problem are established by considering the dependence-diagram among these variables; then, the synthesis and loop-closure equations of the mechanism under study are analytically obtained in order to pose the necessary equations included in the objective function; finally, the minimization of the objective function is solved. The latter step can be done in an analytic way, in some simple cases, or numerically for more complex designs.

Initially, the dependence-diagram among existing variables is established (see Fig. 1). The $\vec{s}$ variables are:

- On the one hand, the independent variables of the synthesis: the degrees of freedom of the mechanism, $\vec{e}$, and the design parameters, $\vec{a}$.

- On the other hand, the variables involved in the loop-closure equations, $\vec{l}$, have to be also included, which depend on the degrees of freedom of the mechanism and the design parameters. Thus, we have: $\vec{l}=\vec{l}(\vec{e}, \vec{a})$.

Accordingly, the general dependence-diagram of the synthesis variables for a certain mechanism can be posed as it is shown in Fig. 1.

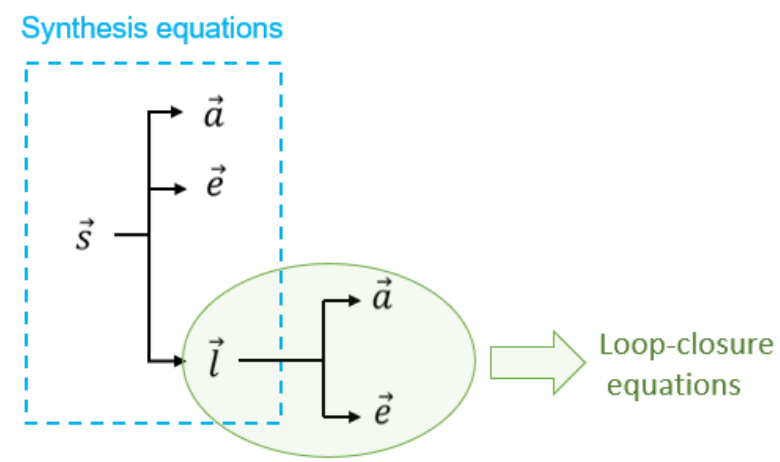

Fig. 1. Dependence-diagram among synthesis variables.

Focusing on path generation, the purpose of the optimum dimensional synthesis is to find the most adequate design capable of tracing a trajectory as similar as possible to a prescribed one. In a planar case, the prescribed or desired trajectory will be formed by a set of $P_{i}^{d}$ desired points so that the trajectory is defined by a set of $\left(x_{i}^{d}, y_{i}^{d}\right)$ coordinates, $\forall i=1,2, \ldots N$. As an illustrative example, the four-bar mechanism shown in Fig. 2 will be used. In the figure, the $a_{j}$ design parameters, the $\varphi$ input (the degree of freedom), and the variables of the loop-closure equations, $(\theta, \psi)$, are established, together with a certain desired trajectory and the real generated trajectory of the mechanism. 


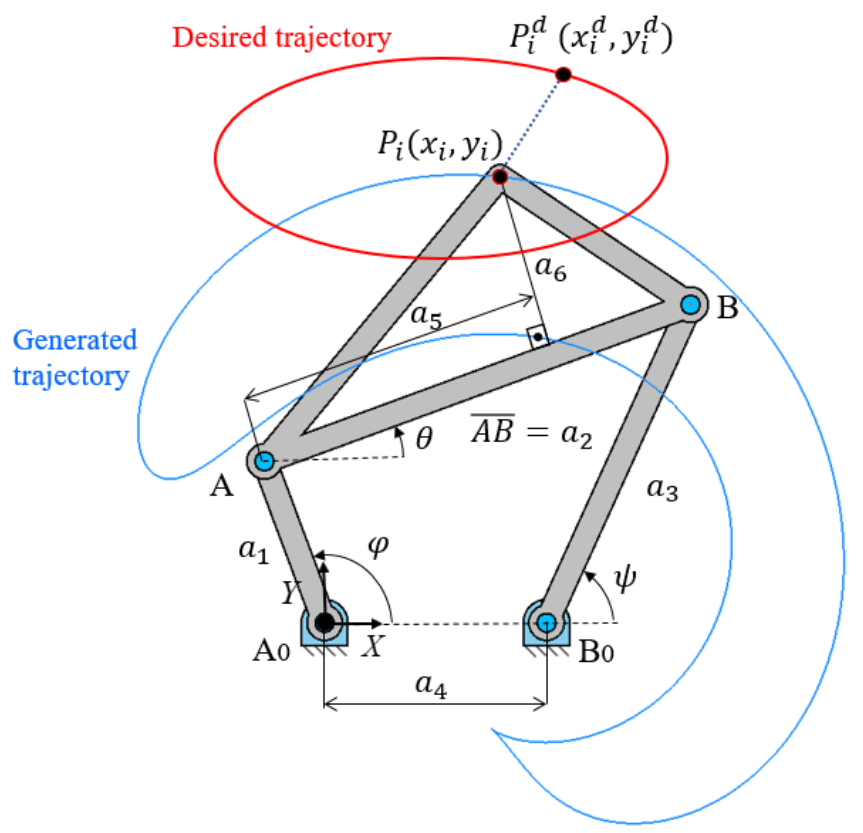

Fig. 2. Four-bar mechanism. Desired and real generated trajectory

The error function, $E$, can be defined as the sum of the squared distance between the generated points, $P_{i}$, and the desired points, $P_{i}^{d}$. Thus:

$$
E=\sum_{i=1}^{N}\left[\left(x_{i}-x_{i}^{d}\right)^{2}+\left(y_{i}-y_{i}^{d}\right)^{2}\right]
$$

The equation system that has to be solved results from the minimization of the error function. Accordingly:

$$
\frac{\partial E}{\partial a_{j}}=0 \rightarrow \sum_{i=1}^{N}\left[\left(x_{i}-x_{i}^{d}\right) \frac{\partial x_{i}}{\partial a_{j}}+\left(y_{i}-y_{i}^{d}\right) \frac{\partial y_{i}}{\partial a_{j}}\right]=0
$$

$\forall j=1,2, \ldots, n$ design parameters of the mechanism.

The partial derivatives of Eq. (2), $\frac{\partial x_{i}}{\partial a_{j}}$ and $\frac{\partial y_{i}}{\partial a_{j}}$, are obtained by considering the dependence-diagram among variables established in Fig. 1. For that, the synthesis equations and the loop-closure equations have to be analytically obtained. 
Following with the four-bar mechanism, the synthesis equations $x=f_{x}\left(\theta, \psi, \varphi, a_{j}\right)$ and $y=f_{y}\left(\theta, \psi, \varphi, a_{j}\right)$ yield:

$$
\begin{aligned}
& x=a_{1} \cos \varphi+a_{5} \cos \theta-a_{6} \sin \theta \\
& y=a_{1} \sin \varphi+a_{5} \sin \theta+a_{6} \cos \theta
\end{aligned}
$$

And the loop-closure equations are:

$$
\begin{gathered}
a_{1} \cos \varphi+a_{2} \cos \theta=a_{4}+a_{3} \cos \psi \\
a_{1} \sin \varphi+a_{2} \sin \theta=a_{3} \sin \psi
\end{gathered}
$$

Hence, the partial derivatives to be obtained are:

$$
\begin{aligned}
& \frac{\partial x}{\partial a_{j}}=\frac{\partial f_{x}}{\partial \theta} \cdot \frac{\partial \theta}{\partial a_{j}}+\frac{\partial f_{x}}{\partial \psi} \cdot \frac{\partial \psi}{\partial a_{j}}+\frac{\partial f_{x}}{\partial a_{j}} \\
& \frac{\partial y}{\partial a_{j}}=\frac{\partial f_{y}}{\partial \theta} \cdot \frac{\partial \theta}{\partial a_{j}}+\frac{\partial f_{y}}{\partial \psi} \cdot \frac{\partial \psi}{\partial a_{j}}+\frac{\partial f_{y}}{\partial a_{j}}
\end{aligned}
$$

Note that the partial derivatives $\frac{\partial \theta}{\partial a_{j}}$ and $\frac{\partial \psi}{\partial a_{j}}$ can be implicitly obtained through the loopclosure equations.

In many cases, the value of the input is prescribed for each $P_{i}^{d}$ point. This is known in the literature as prescribed timing. However, this often implies a restriction in the optimization process. On the contrary, if the input values are included in the set of optimization variables, the optimum input parameters so that the mechanism best fits the desired trajectory can be achieved. This latter option, called unprescribed timing, has been integrated in the presented optimization procedure. Considering unprescribed timing, the set of $a_{j}$ optimization variables include both the design parameters and the input parameters.

\section{Understanding optimum synthesis using GIMSYNT software}

The optimum synthesis approach has been programmed in Matlab for the four-bar mechanism and the slider-crank mechanism, and it serves as an educational tool for students. A GUI (Guide User Interface) has been created, resulting in the software named GIMSYNT, its main window for the case of the four-bar mechanism being shown in Fig. 3. 


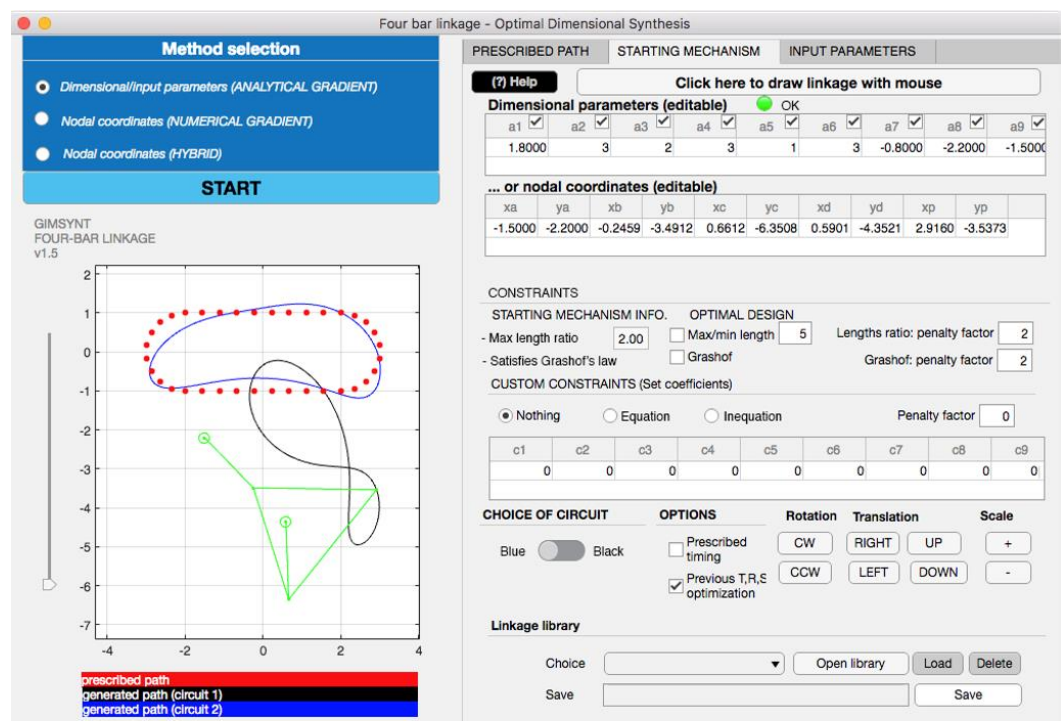

Fig. 3. Main window of GIMSYNT software

The basic guidelines of the procedure have been implemented in the software, together with some additional options:

- A library of several designs which trace different trajectories. This enables the student to select the most appropriate starting mechanism.

- Possibility of applying an initial phase of Translation-Rotation-Scaling (TRS) to better adapt the initial design to the desired trajectory.

- Selection of the initial approximation points by writing the corresponding $\left(x_{i}, y_{i}\right)$ coordinates or by simply clicking on the points of the starting trajectory.

- Possibility of selecting the design parameters that will be optimized. The student can also activate the option of unprescribed timing.

- Include design constraints such as: limited ratio between maximum and minimum length, choice of fulfilling the Grashof criterion, establishing the maximum length of a certain element, and so on.

With the purpose of explaining how these latter options influence on the optimization results, three different cases will be compared. In all the three examples shown in subsequent figures, the desired trajectory is depicted in red color, the initial trajectory of the starting mechanism is plotted in green, and the generated trajectory of the optimum design is depicted in blue. 


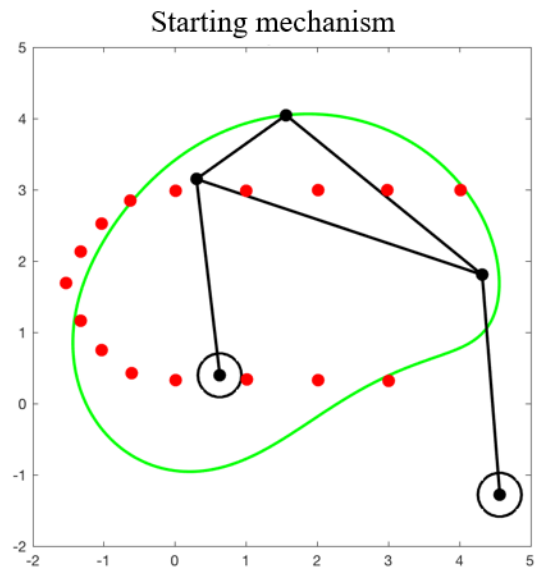

(a)

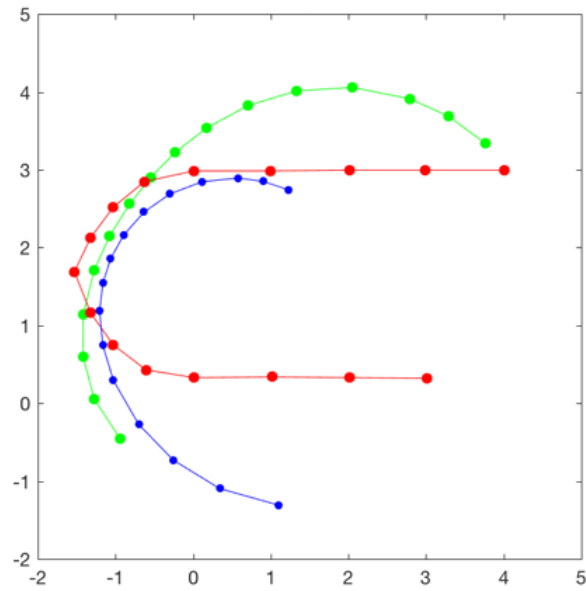

(c)

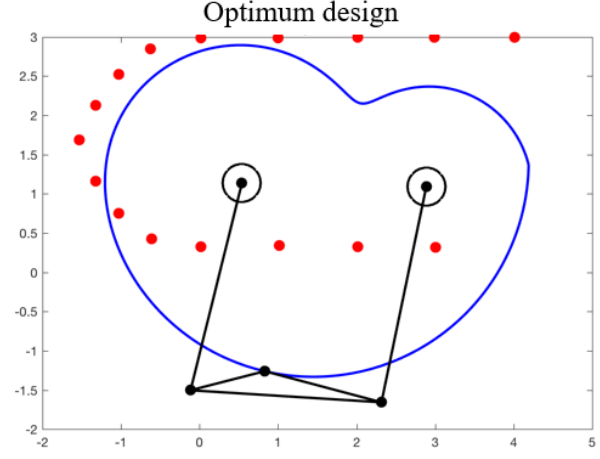

(b)

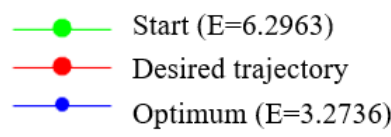

Fig. 4. Case 1: (a) Starting and (b) optimum mechanism; (c) Visualizing trajectories among precision points

The first example, Case 1, starts from a quite suitable starting mechanism because, as it can be observed in Fig. 4a, the initial trajectory resembles the desired trajectory. This starting mechanism has been selected from the implemented library. However, for the Case 1, the unprescribed timing option is not activated meaning that the input parameters must remain constant. This greatly limits the optimization process and the final result, that is, the optimum design, does not generate an acceptable trajectory. The resulting trajectory is shown in blue color in Fig. 4b and Fig. 4c, and the error between the desired and generated trajectory, given by Eq. (1), is 3.2736 units.

In Case 2, represented in Fig. 5, different actions have been realized to enhance the optimization results in comparison to the previous case. On the one hand, the initial 
starting mechanism traces a trajectory more similar to the desired one. Besides, before applying the optimization tactic, a previous phase of TRS adapts the starting design to get even closer to the desired path. As it can be observed in Fig. 5c, the TRS transformation obtains the black trajectory which is indeed much better than the initial one (in green) and quite similar to the desired one (in red). On the other hand, the option of unprescribed timing is included, so that the optimum input values are also obtained, together with the optimum design parameters. The final optimum design is shown in Fig. 5b, having a really small error of 0.00149 units.

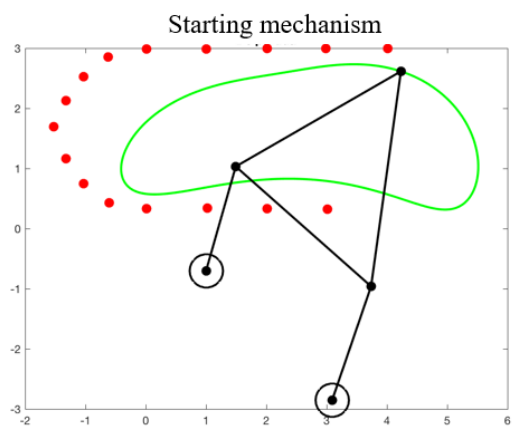

(a)

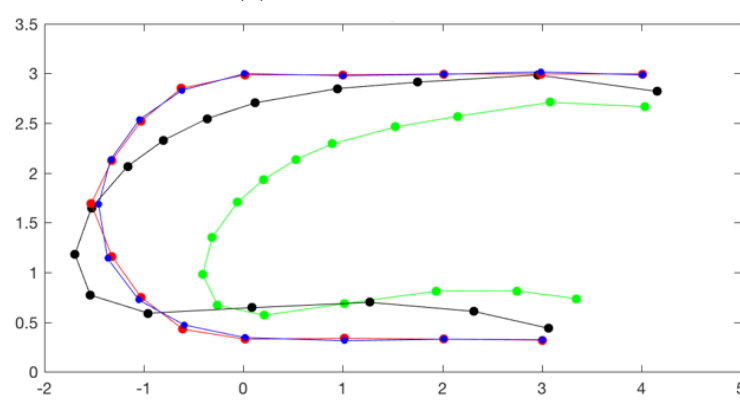

(c)

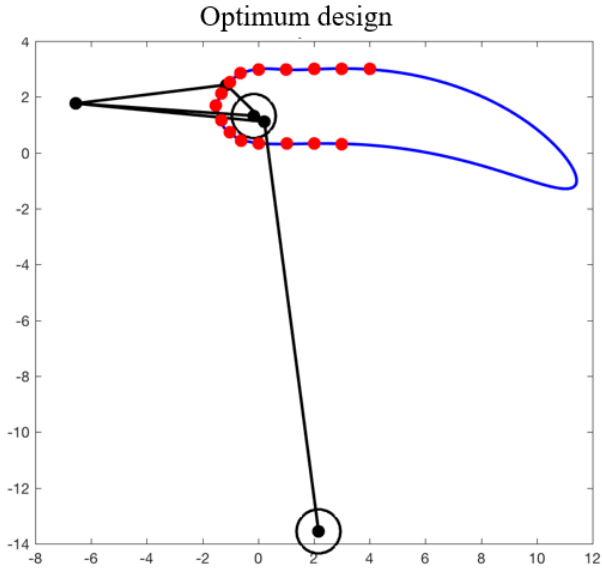

(b)

Fig. 5. Case 2: (a) Starting and (b) optimum mechanism; (c) Visualizing trajectories among precision points

Despite achieving very good results in Case 2, concerning the existing small error between the generated and the desired path, it can be observed in Fig. 5b that, in the optimum design which has been achieved, the size of one of its bars is out of proportion to 
the remaining bars. In practice, this design could not be valid. As it has been previously mentioned, different design constraints can be included in the optimization approach. This has been done in Case 3 which is shown in Fig. 6. In this case, a limitation of the ratio between maximum and minimum lengths is imposed by integrating a penalty function in the optimization process. Consequently, as it can be observed in Fig. 6b and Fig. $6 \mathrm{c}$, the resulting error is not so small as the one achieved in Case 2 (though it is rather satisfactory), but the achieved design is much more adequate in practice.

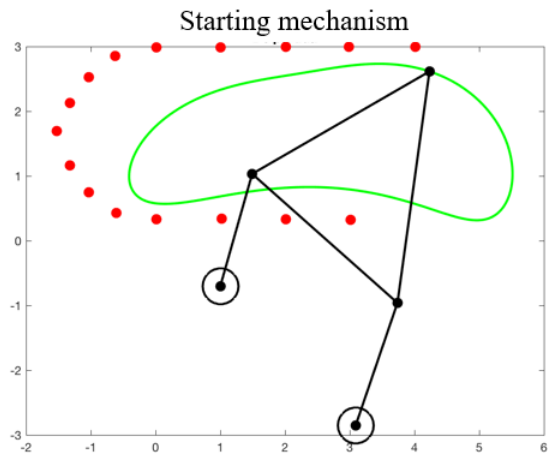

(a)

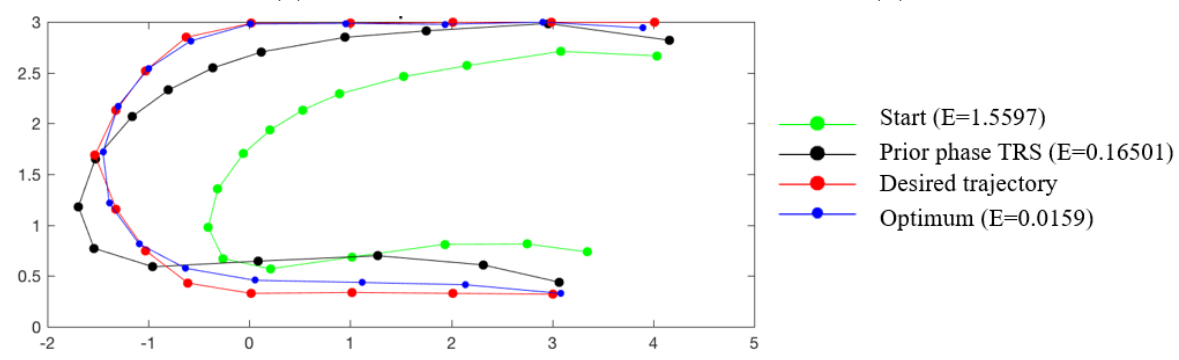

(c)

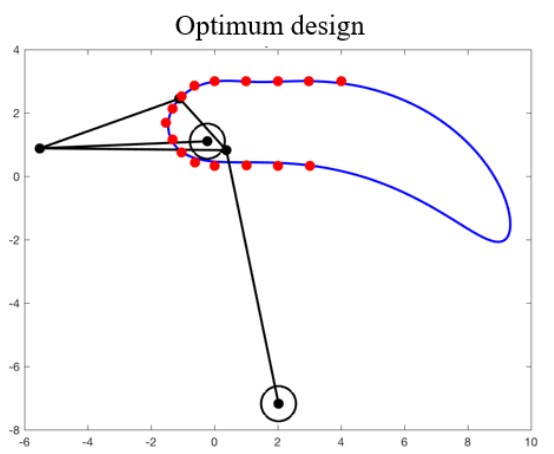

(b)

Fig. 6. Case 3: (a) Starting and (b) optimum mechanism; (c) Visualizing trajectories among precision points

\section{Conclusions}

The optimum dimensional synthesis approach presented in this paper has been mainly developed with the intention of being a comprehensive and didactic procedure to help students to really understand how the optimization process works. The guidelines of this procedure can be programmed by the students for a certain case under study, indeed, they do it during some of the practical sessions of a related subject taught in the Master of Mechanical Engineering. In addition to this, GIMSYNT software has proven to be very useful to compare different designs and achieve a suitable optimum final design. Specially, the understanding of the influence of the different options that can be incorporated in the optimization process stands out, such as the importance of the 
starting mechanism, the feasibility of applying the unprescribed timing approach and the benefits of including an initial TRS transformation.

\section{Acknowledgments}

The authors wish to acknowledge the financial support received from the Spanish Government through the Ministerio de Economía y Competitividad (Project DPI201567626-P (MINECO/FEDER, UE)), and the financial support given to the research group, through the project with Ref. IT949-16, given by the Departamento de Educación, Política Lingüística y Cultura of the Regional Government of the Basque Country.

\section{References}

1. Petuya, V., Macho, E., Altuzarra, O., Pinto, C., Hernández, A: Educational Software Tools for the Kinematic Analysis of Mechanisms. Computer Applications in Engineering Education, Vol. 22, pp. $72-86$ (2014).

2. Han, C.-Y.: A general method for the optimum design of mechanisms. Journal of Mechanisms, Vol. 1, pp. 301-313 (1996).

3. Sancibrián, R., Juan, A., Sedano A., Iglesias, M., García, P., Viadero, F., Fernández, A.: Optimal Dimensional Synthesis of Linkages Using Exact Jacobian Determination in the SQP Algorithm. Mechanics Based Design of Structures and Machines, Vo. 40, pp. 469-486 (2012).

4. Avilés, R., Navalpotro, S., Amezua, E., Hernández, A.: An energy-based general method for the optimum synthesis of mechanisms. Journal of Mechanical Design, Vol. 116, No 1, pp. 127-136 (1994).

5. Cabrera, J.A., Ortiz, A., Nadal, F., Castillo, J.J.: An evolutionary algorithm for path synthesis of mechanisms. Mechanism and Machine Theory, Vol. 46, pp. 127-141 (2011).

6. Khroshidi, M., Soheilypour, M., Peyro, M., Atai, A., Shariat, M.: Optimal design of four-bar mechanisms using a hybrid multi-objective GA with adaptive local search. Mechanism and Machine Theory, Vol. 46, pp. 1453-1465 (2011).

7. Sánchez, F. T., Pérez, A.: Global optimization in path synthesis based on design space reduction. Mechanism and Machine Theory, Vol. 38, pp. 579-594 (2003).

8. Singh, R., Chaudhary, H., Singh, A.: Defect-free optimal synthesis of crank-rocker linkage using nature-inspired optimization algorithms. Mechanism and Machine Theory, Vol. 116, pp. 105-122 (2017).

9. Kafash, S. H., Nahvi, A.: Optimal synthesis of four-bar path generator linkages using Circular Proximity Function. Mechanism and Machine Theory, Vol. 115, pp. 1834 (2017). 